\title{
Interorgan communication by exosomes, adipose tissue, and adiponectin in metabolic syndrome
}

\author{
Shunbun Kita, ${ }^{1,2}$ Norikazu Maeda, ${ }^{1,3}$ and lichiro Shimomura ${ }^{1}$ \\ 'Department of Metabolic Medicine, 'Department of Adipose Management, and ${ }^{3}$ Department of Metabolism and Atherosclerosis, Craduate School of Medicine, Osaka University, Osaka, Japan.
}

\begin{abstract}
Adipose tissue plays important roles in regulating whole-body energy metabolism through its storage function in white adipocytes and its dissipating function in brown and beige adipocytes. Adipose tissue also produces a variety of secreted factors called adipocytokines, including leptin and adiponectin. Furthermore, recent studies have suggested the important roles of extracellular vesicles of endosomal origin termed exosomes, which are secreted from adipocytes and other cells in adipose tissue and influence whole-body glucose and lipid metabolism. Adiponectin is known to be a pleiotropic organ-protective protein that is exclusively produced by adipocytes and decreased in obesity. Adiponectin accumulates in tissues such as heart, muscle, and vascular endothelium through binding with T-cadherin, a glycosylphosphatidylinositol-anchored (CPI-anchored) cadherin. Recently, adiponectin was found to enhance exosome biogenesis and secretion, leading to a decrease in cellular ceramides, excess of which is known to cause insulin resistance and cardiovascular disease phenotypes. These findings support the hypothesis that adipose tissue metabolism systemically regulates exosome production and whole-body metabolism through exosomes. This review focuses on intra-adipose and interorgan communication by exosomes, adiponectin-stimulated exosome production, and their dysregulation in metabolic diseases.
\end{abstract}

\section{Introduction}

Obesity is associated with an array of complications, including cardiovascular diseases, type 2 diabetes (T2D), fatty liver disease, osteoporosis, airway disease, degenerative disorders including dementia, and certain cancers. In fact, obesity accompanied 4.72 million deaths and 148 million disability-adjusted life-years globally in 2017. It was the fourth leading risk factor for mortality $(1,2)$. These complications are considered to occur in large part as a result of insulin resistance induced by obesity. Important to note is the notion that adipose tissue is not only an energy storage organ but also an endocrine organ, secreting factors that affect the function of cells and tissues/organs throughout the body. Overproduction and/or imbalance of adipose secretome, i.e., overproduction of proinflammatory adipocytokines and diminished expression of antiinflammatory adipocytokines as occurs in obesity are considered to account for systemic metabolic dysfunction and cardiovascular disease (3). In addition to such dysregulation of adipocytokines, exosomes secreted from adipocytes and other cells in adipose tissue have been revealed to be altered in obesity (4-7). Exosomes are vesicles that envelop intracellular molecules including microRNAs, bioactive lipids, and proteins $(8,9)$. Their potential roles in cell-to-cell communication position them as previously unrecognized and important endocrine machinery.

Among the various adipocytokines, adiponectin exists in plasma at the highest circulating concentration (10-13). It circulates at between 3 and $30 \mu \mathrm{g} / \mathrm{mL}$ in plasma, corresponding to molec-

Conflict of interest: The authors have declared that no conflict of interest exists. Copyright: (c) 2019, American Society for Clinical Investigation.

Reference information: J Clin Invest. 2019;129(10):4041-4049.

https://doi.org/10.1172/JCl129193. ular quantities that are $10^{3}$ - to $10^{6}$-fold higher than ordinary hormones and cytokines. Since its discovery as the most abundant adipose secretory protein around 20 years ago (10-13), adiponectin has appeared in the title of over 7,000 reports. However, two very important questions remained unanswered until now: (i) Why is such an abundant concentration of adiponectin in plasma required? (ii) Why are multimeric conformations required for adiponectin to exert its functions?

Several recent studies have reported that $\mathrm{T}$-cadherin mediates cardiovascular protection and skeletal muscle regeneration via the clinically important high molecular weight (HMW) multimeric adiponectin (14-17). T-cadherin facilitates adiponectin accumulation in multivesicular bodies, a subtype of endosome in which exosomes are generated, wherein adiponectin stimulates exosome biogenesis and secretion (17-19).

In this Review, we will discuss adipose tissue exosomes, their roles as signaling packages and waste-disposing vesicles, and further, their roles in glucose and lipid metabolism and adiponectin functions in exosomes. We also discuss the current technological limitations of studying exosome and future perspectives.

\section{Adipose tissues secrete adipocytokines and exosomes}

Adipose tissues secrete exosomes, influencing whole-body metabolism. It is recognized that adipose tissue is the largest endocrine organ, secreting over 600 proteins (20-22), including the first-discovered leptin (23) and the subsequently described adiponectin (10-13). To date, numerous adipocyte-derived secretory factors have been identified that play a role in the maintenance of glucose, lipid, and energy homeostasis, each of them contributing to communication 
Table 1. Evolutionary view of adipose tissue-, exosome-, and adiponectin-related proteins

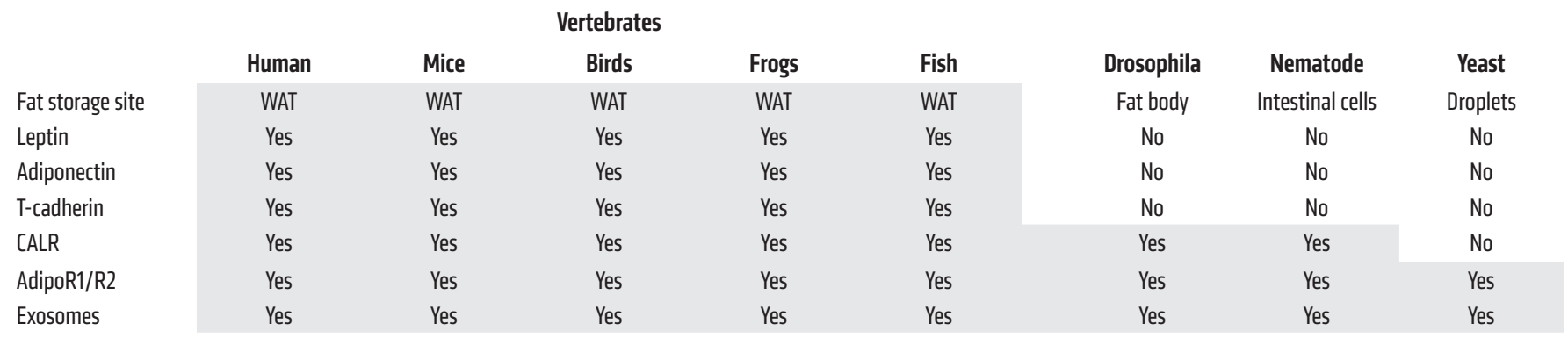

Energy storage in the form of triglycerides in droplets is an evolutionarily conserved way to keep energy in the form of biologically inactive material in a separate repository for future needs (26). For example, lipid droplets are conserved from yeast to mammals (136), and the droplet-stabilizing perilipin family (PLIN family) protein also exists in yeast (137) and Caenorhabditis elegans (138). Fat is stored in the intestine in C. elegans, a defined fat body in Drosophila. Sharks lack adipose tissue and use their liver to store fat, although their genomes have homologs of leptin and adiponectin. Exosomes, too, are conserved among eukaryotes and produced in essentially all cells throughout the body (27). WAT becomes apparent in vertebrate species, coinciding with the appearance of leptin, adiponectin, and T-cadherin $(26,139)$. APN, adiponectin; T-cad, T-cadherin; calr, calreticulin. The table is adapted in large part from a previous publication (26), combined with knowledge based on genetic information from the NCBI Gene database.

within adipose tissues and/or interorgan communication between adipose tissue and other tissues involved in the maintenance of metabolic homeostasis, such as muscle, heart, liver, islets, kidney, and even brain. Moreover, recent understanding of the secretory role of adipose tissues went beyond such secreted proteins. Thomou et al. reported that the majority of circulating microRNAs included in exosomes are derived from adipocytes and travel to the liver, where they improve glucose tolerance and reduce hepatic FGF21 expression (7). Crewe et al. demonstrated exosomal communication between endothelial cells and adipocytes in adipose tissues (24). They elaborately isolated adipose-tissue extracellular vesicles and showed that proteins and lipids capable of modulating cellular signaling pathways were involved in such small vesicles (24). Flaherty et al. reported that adipocytes can release neutral lipids directly via exosomes in addition to the lipolysis reaction, which releases fatty acids and glycerol through the action of neutral lipases (4). Ying et al. demonstrated that exosomal microRNAs derived from adipose tissue macrophages regulate whole-body glucose metabolism by modulating adipocyte functions (6). Pan et al. reported that adipocyte-secreted exosomal microRNA-34a inhibits M2 macrophage polarization to promote obesity-induced adipose inflammation (5). Together, these studies support the broad influence of adipose tissue-derived exosomes in whole-body metabolism.

Evolutionary view of adipose tissue, exosomes, and adipocytokines. Energy storage in the form of triglycerides is a conserved way to store energy for future needs. Although sharks store fat primarily in the liver (25), a tissue of endodermal origin, most vertebrate species store fat primarily in white adipose tissue (WAT), a tissue of mesodermal origin (26) (Table 1). From an evolutionary viewpoint, WAT's appearance in vertebrates accompanied a dramatic change in organ fat storage, from simple fat storage to an organ that secretes adipocytokines like leptin and adiponectin (Table 1). However, exosomes, defined as secreted vesicles generated in late-endosomal compartments, are conserved among eukaryotes (27).

A striking difference between mammals and other vertebrate animals is their diverse distribution of WAT in many areas in the body, including intra-abdominal depots around the visceral organs, intestines, and perirenal areas, as well as in subcutaneous depots in the buttocks, thighs, and abdomen (26). In mammals, the adipose organ is composed not only of white adipocytes, which are the primary site of energy storage, but also brown adipocytes, which are important in energy expenditure in the form of thermogenesis. Adiponectin is expressed, in this sense, throughout the body, irrespective of adipose tissue color, i.e., in every adipocyte in all areas, including in beige/brown adipocytes, in ectopic adipocytes that are occasionally observed in degenerating tissues, and in bone marrow adipocytes $(28,29)$. Such ubiquitous expression of adiponectin in all adipocytes lets the adiponectin promoter be exploited to target adipocytespecific expression of genes of interest, using Cre recombinase or other methods, to produce adipocyte-specific transgenic mice.

Three types of adiponectin binding partners have been suggested: adiponectin receptors (adipoRs), calreticulin, and T-cadherin. From an evolutionary viewpoint, WAT's appearance in vertebrates accompanied the emergence of both adiponectin and T-cadherin (Table 1).

\section{Exosomes are multifunctional in glucose and lipid metabolism}

Exosome biogenesis and secretion. Exosomes initially form as intraluminal vesicles (ILVs), which are generated by inward budding of the limiting membrane during endosome maturation into multivesicular bodies (MVBs) in the endocytic pathway (30). The endosomal sorting complexes required for transport (ESCRT-0, -I, -II, -III) and the associated ATPase Vps 4 complex play important roles in this process. However, after depletion of key subunits of all four ESCRTs, ESCRT-independent ILV biogenesis still exists in mammalian cells. MVBs can fuse either with lysosomes for intracellular degradation or with the plasma membrane, resulting in release of ILVs into the extracellular space as exosomes (27).

Exosome biogenesis and/or release is affected by various molecules, including the ESCRT machinery components, Rab GTPases acting on vesicular traffic, membrane-spanning tetra- 


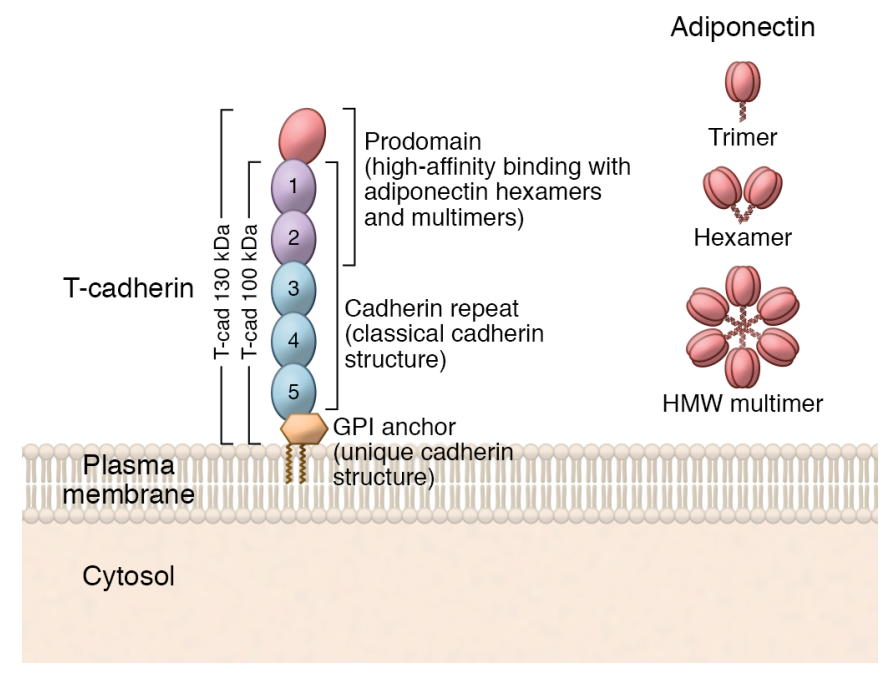

Figure 1. Structure of T-cadherin. T-cadherin is a unique GPI-anchored cadherin. It is classified as a member of the classical cadherins, but it lacks C-terminal transmembrane and cytosolic domains (106). T-cadherin exists both on the plasma membrane and in endosomes, including multivesicular bodies where exosomes are generated (18). T-cadherin was discovered as an adiponectin binding partner by evaluating cell binding to coated recombinant adiponectin produced in HEK293 mammalian cells (57). Its unique prodomain gives T-cadherin high-affinity interaction with native HMW adiponectin in serum (19).

spanins, and the intracellular adaptor syntenin (31). Features of cellular metabolic status such as ceramide metabolism, ER stress, autophagy, and intracellular calcium also affect exosome biogenesis and/or secretion. Adiponectin, a protein secreted exclusively from adipocytes, stimulates exosome biogenesis in endothelial and skeletal muscle cells expressing T-cadherin (18). Crewe et al. discovered that glucagon regulates exosome production from endothelial cells in adipose tissues (24). These findings indicate that exosome biogenesis is a metabolically regulated process in addition to being a process that impacts metabolism.

Exosomes function as signaling packages. Exosomes are small vesicles $(30-100 \mathrm{~nm})$ delimited by a lipid bilayer and are thought functionally to mediate cell-to-cell communication under normal and pathological conditions by transferring active proteins, mRNA, and small noncoding RNAs stably in various biofluids (8, 9). In this regard, a recent study reported that adipose-derived circulating microRNAs in exosomes regulate important metabolic gene expression in distant tissues such as liver (7). DICER cleaves double-stranded RNA (dsRNA) and pre-microRNA into short, single-stranded RNA fragments called small interfering RNA (siRNA) and microRNA, respectively. The study by Thomou et al. found that circulating microRNA content was decreased in adipocyte-specific Dicer-KO mice, suggesting that adipocyte-secreted exosomes occupy the majority of circulating exosomes (7). In contrast, however, using adipocyte-specific expression of the fluorescent protein tdTomato in mice, Flaherty et al. reported that adipocyte-derived exosomes represent a minority of the exosomes in the circulation under normal conditions (4). Despite these apparently conflicting findings, a number of studies (discussed below) support the importance of exosome-derived signals from adipose tissue in energy homeostasis and metabolism.
In clinical studies, it was reported that exosomal protein and microRNA profiles were altered in T2D patients $(32,33)$. In accordance with these human data, it was reported that adipose tissue macrophage-derived exosomes affected insulin sensitivity in mice (6). Exosomes from adipose tissue macrophages in obese mice caused glucose intolerance and insulin resistance when administered to lean mice (6). In turn, exosomes from adipose tissue macrophages in lean mice improved glucose intolerance and insulin resistance when administered to obese mice (6). The changes observed in microRNAs involved in exosomes, such as miR-155, were suggested to have important roles in regulating insulin sensitivity and glucose homeostasis (6). Moreover, an obesity-associated increase of exosomal microRNA-34a secretion from adipocytes inhibited M2 macrophage polarization and promoted obesity-induced adipose inflammation (5). The expression of miR-34a in adipose tissues also progressively increased with the development of diet-induced obesity in mice. Adipose- or adipocyte-specific miR-34a-KO mice were resistant to obesity-induced glucose intolerance, insulin resistance, and systemic inflammation, and this was accompanied by antiinflammatory M2 polarization of adipose-resident macrophages from the proinflammatory M1 phenotype (5). Taken together, these findings suggest the important roles of exosomal transfer of microRNAs for the regulation of glucose and energy metabolism (4-7).

On the other hand, a quantitative assessment of the microRNA content of extracellular vesicles using a stoichiometric approach showed that microRNA copy number per vesicle in a blood sample was less than 1, even for the most abundant microRNAs, suggesting that most individual exosomes do not carry biologically significant numbers of microRNA and are unlikely to function as vehicles for microRNA-based communication (34). Because an exosome is a package of multiple bioactive proteins, lipids, and nucleic acids including microRNAs, its function in the recipient cells might be based on the orchestration of a variety of signals.

A recent report noted increased particle numbers in fasting plasma of T2D patients versus euglycemic controls (35). Quantification of exosomes in biological fluids is still technically challenging $(36,37)$. Polyethylene glycol-mediated precipitation methods like ExoQuick used in this study precipitate essentially all large molecules, including lipoproteins and aggregated proteins, in addition to exosomes and microvesicles. Problematically, nanoparticle tracking analysis (NTA) cannot discriminate them; it can only evaluate the size distribution of particles in test fluids. A simple, semiquantitative method was developed to measure exosomes in blood based on the combination of polyethylene glycol-based purification, standard differential ultracentrifugation techniques, and Western blotting $(18,38)$. Although it is semiquantitative, this method can measure a number of exosome components simultaneously and may give information on changes in some important exosome cargos induced by metabolic challenges.

Recently, adipose tissue endothelial cells were reported to produce high amounts of exosomes in response to glucagon (24). This study established the importance of exosome-mediated intra-adipose and interorgan communications in energy metabolism. Exosomes secreted from adipose endothelial cells travel to neighboring adipocytes, and reciprocally, adipocyte-secreted exosomes travel to endothelial cells. These exosomes can be taken up by neighboring macrophages in adipose tissue, and are 


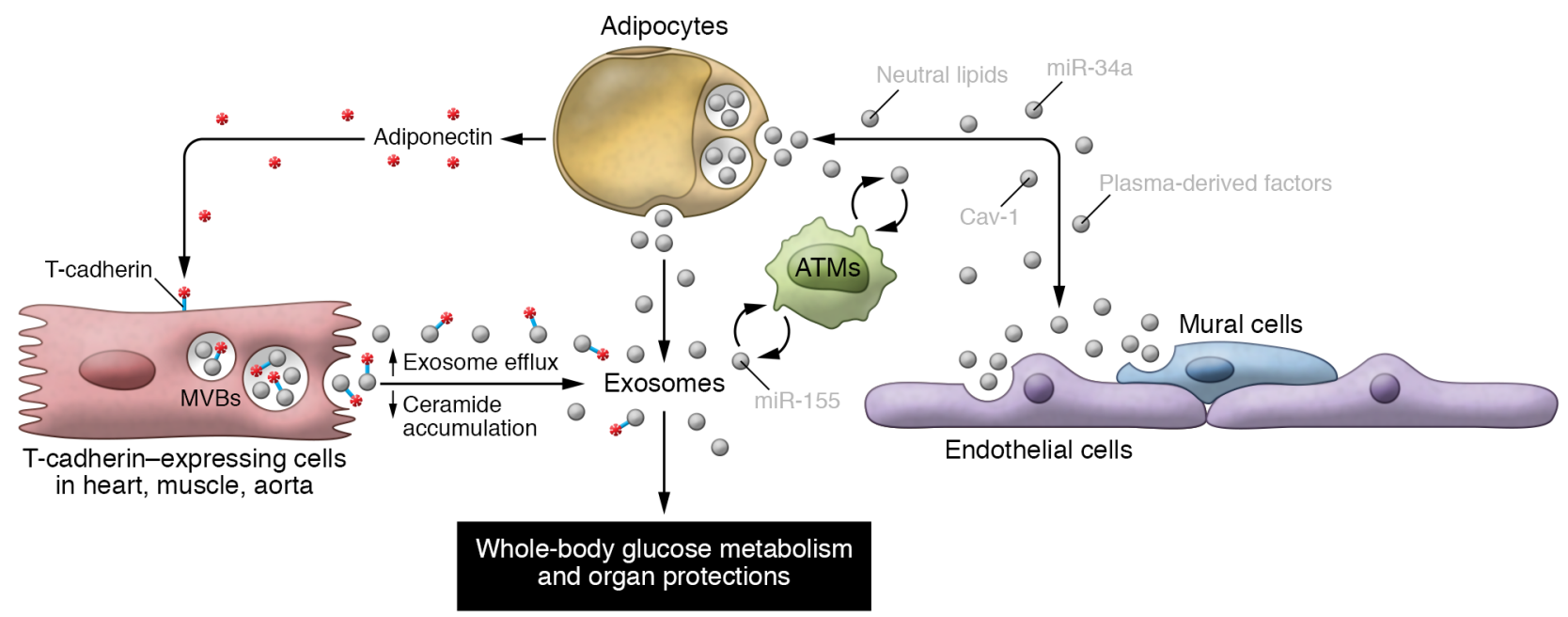

Figure 2. Exosome-mediated transfer of signals and substances controls whole-body glucose metabolism. Various cell types communicate with each other by transferring signals, such as microRNAs, and substances, such as neutral lipids in adipose tissue (4-6, 24). Macrophages phagocytose and secrete exosomes. Adipose tissue-derived exosomes affect whole-body glucose metabolism (6, 7). Adiponectin secreted from adipocytes stimulates exosome production from T-cadherin-expressing cells in aorta, heart, and muscle and affects systemic exosome levels (17-19). The adiponectin-mediated production of exosomes also may also affect whole-body glucose metabolism.

rich in proteins and lipids that are capable of modulating cellular signaling pathways (24). In agreement with this notion, adipocytederived exosomes containing large amounts of lipids induced an adipose tissue macrophage-like phenotype in bone marrow cells in cell culture (4). It is noteworthy that these two studies elaborately developed new methods to isolate adipose tissue extracellular vesicles (24) and adipocyte-derived exosomes (4), respectively. Moreover, these novel and fundamental notions might challenge the long-believed "adipose chronic inflammation theory" that is based on observations that increased lipolysis activates macrophages. Therefore, it may be reasonable to speculate that metabolically stressed adipocytes in obesity may secrete exosomes bearing information that triggers low-grade inflammation, thus causing adipose inflammation and insulin resistance.

Exosomes function as waste disposal bags. In addition to the above-mentioned signaling role of exosomes, exosomal release serves as an alternate disposal pathway to lysosomes (39). Misfolded proteins or several aggregated toxic proteins, such as amyloid $\beta$ (40), tau (41), and prion protein (42), were shown to be released in exosomes. A more recent study revealed that a cell's exosomal release suppresses its own cellular senescence by excreting accumulated harmful cytoplasmic damaged DNA (43). These observations suggest that a cell's exosomal release can attenuate its own cellular stress and maintain its homeostasis by exporting various unnecessary or harmful materials.

Although the fate of secreted exosomes and their contents is important, the molecular basis for their active targeting to acceptor cells is not well understood. For example, it is reported that integrins on exosomes can interact with adhesion molecules and matrix proteins on acceptor cells, thus enabling active targeting (44). How bound exosomes release their content, i.e., microRNAs and bioactive proteins, into the cytosol of the recipient cells for signaling is poorly understood. Recent studies suggested the possible existence of a mechanism by which RNA and other exosome cargos can escape the intrinsically degradative endosomal pathway (45-47).
On the other hand, the majority of exosomes can be phagocytosed by macrophages (48-51). Exosomes have no intrinsic ATP production and expose phosphatidylserine on their surface, similarly to apoptotic cells, thus enabling their recognition by phagocytes like macrophages $(49,51-53)$. Phagocytosed exosomes travel into late endosomes, including MVBs, and large parts can be degraded by lysosomes in macrophages, which have more degradative activity than other cells. Interestingly, adipocyte-derived exosomes were found to contain large amounts of neutral lipids and induce lipidladen macrophages in adipose tissue (4). Such lipid droplets in macrophages are catabolized, not by an autophagy-related pathway but by an endocytosis-related pathway, suggesting that the droplets are not generated within macrophages but rather are endocytosed into macrophages $(4,54)$. Thus, the tissue-specific phenotype and function of macrophages in adipose tissue may be induced by fatty acids generated in lysosomes from such neutral lipids transferred from adipocytes via these atypical exosomes.

\section{Adiponectin regulates systemic exosome level through T-cadherin}

There have been three classes of adiponectin receptor reported: adipoRs (55), calreticulin (56), and T-cadherin (57). Although adiponectin was reported to exert metabolically important roles through adipoRs (58-60) and immunologically important roles through calreticulin (57), our review will primarily focus on T-cadherin because of the large body of evidence supporting its modulation of adipose exosomal communication.

Adiponectin circulates in plasma in three major forms: trimer, hexamer, and HMW multimer (61-63). The HMW multimeric adiponectin has been shown to be the more active form of adiponectin in improving insulin sensitivity and protecting against glucose intolerance (64-67). Impaired multimerization or selective reduction of HMW multimeric adiponectin concentrations in plasma was also associated with various metabolic diseases such as obesity, insulin resistance, T2D, and arteriosclerosis (67-69). Increase 
in the ratio of HMW multimeric adiponectin to total adiponectin was more closely associated with improved insulin sensitivity in diabetic mice and PPAR $\gamma$ agonist-treated diabetic patients than total adiponectin (69), further supporting the HMW multimer's physiological relevance.

Obesity, aging, and heart failure regulate plasma adiponectin. HMW octadecameric adiponectin exceeds a molecular size of $450 \mathrm{kDa}(70)$. After secretion from adipocytes into the adipose interstitial fluid, this large multimeric adiponectin presumably cannot pass through the capillary wall into circulation; rather, it is likely to enter the lymph from interstitial fluid (71). Because lymph transport takes longer and variable time to enter into circulation relative to capillary transcytosis, this notion is compatible with the observation of relatively little diurnal change in plasma adiponectin concentration in human subjects (72), despite the profound change in expression and secretion seen in cultured adipocytes following metabolic challenges $(10,73)$.

Once secreted from adipocytes, there seems to be no active interconversion between molecular species of adiponectin in plasma (61). The most striking feature of this beneficial adipocytokine is its decrease in obesity in human subjects. Adiponectin circulates at the highest plasma concentration known among adipocytokines (32, 74-76). In humans, plasma adiponectin concentration was inversely correlated with body weight and BMI in cross-sectional research $(76,77)$. Regulation of adiponectin's circulating level has not been fully elucidated, although negative-regulation mechanisms have been demonstrated, such as those involving TNF- $\alpha$ (78), IL-6 (79), ROS $(80,81)$, androgen (82), and aldosterone (83, $84)$, all of which were shown to associate with obesity.

Plasma adiponectin level is inversely associated with BMI; on the other hand, it is positively associated with age, even after adjustment for visceral adiposity $(85,86)$. The decrease in its clearance rate in the kidney may be the cause of high levels of adiponectin in the elderly $(87,88)$. In extreme leanness, increased production from increased bone marrow adipose tissue combined with increased production from WAT contributes to hyperadiponectinemia (28). Bone marrow adipose tissue also increases with aging and may account for a higher level of adiponectin in elderly people. On the other hand, C57BL/6J mice, the strain usually used for metabolic experiments, have very few bone marrow adipocytes, especially at young ages (29); thus, they may not be able to represent the changes of adiponectin in plasma associated with aging and obesity. In agreement with this, the well-known inverse association of weight and plasma adiponectin levels in human subjects was not observed consistently in rodent models $(89,90)$.

Several clinical studies have suggested that plasma adiponectin level is an independent prognostic predictor in patients with chronic heart failure ( $\mathrm{CHF})$, naming this the "adiponectin paradox" (48, 91-93). In a prospective study, increased plasma adiponectin predicted poor outcome in future cardiovascular events in patients presenting with stable angina and preserved systolic function (94). Although it is possible that elevated natriuretic peptides in CHF patients may increase adiponectin levels (95), beta blocker use may also play a role in regulating adiponectin $(96,97)$. Interestingly, a decrease in plasma adiponectin concentration was significantly correlated with improvement of cardiac function by beta blocker treatment (96). As we discuss below, organ protec- tion by adiponectin may require T-cadherin and consumption of adiponectin through T-cadherin binding (14-16, 98). Low tissue $\mathrm{T}$-cadherin and/or low T-cadherin-mediated consumption of adiponectin may result in poor $\mathrm{CHF}$ prognosis.

Physiological roles of adiponectin, genetic studies in mice and human subjects. A comprehensive multiethnic analysis of the genetic influences on adiponectin levels was performed and yielded 12 loci, including $A D I P O Q$ (encoding adiponectin) and CDH13 (encoding T-cadherin) (99). A Mendelian randomization approach also tested the hypothesis that adiponectin causally influences insulin resistance and T2D and found no evidence associating adiponectin-lowering alleles with insulin sensitivity and T2D (100), or with coronary heart disease (101). Mendelian randomization has been increasingly used in clinical research and is a powerful tool to help understand mechanisms of disease, especially given the complex metabolic phenomena that commonly occur in human diseases. These studies indicate that SNPs nearby or in the ADIPOQ locus that affect plasma adiponectin level have little impact on insulin sensitivity, diabetes, and cardiovascular disease risk. On the other hand, a multi-loci set of SNPs (liberal approach; gathering all SNPs affecting plasma adiponectin level together) revealed a significant association with cardiovascular disease risks, suggesting that SNPs outside of the ADIPOQ locus that affect plasma adiponectin levels may be important for cardiovascular disease risk (101). Because SNP-associated variation in plasma adiponectin levels in human subjects must be smaller than those induced by a genetic deficiency of adiponectin in mice, animal studies may reveal larger consequences of adiponectin loss. Furthermore, SNPs' small effects on plasma adiponectin levels might be compensated for during life-long periods in human subjects. Indeed, it was reported that the acute depletion of adiponectin resulted in more severe systemic insulin resistance and hyperlipidemia than in mice with congenital loss of adiponectin (102).

Outside of the ADIPOQ locus, the gene locus that most strongly affects plasma adiponectin levels in GWAS is CDH13 (103). Several SNPs in the $C D H 13$ locus are associated with both cardiovascular disease risks and glucose homeostasis $(103,104)$. Importantly, SNPs in the $C D H 13$ locus are also reported to associate with higher plasma adiponectin levels and higher odds ratio for cardiovascular disease risks simultaneously in human subjects $(101,105)$. Loss of $\mathrm{T}$-cadherin in mice significantly increased plasma adiponectin lev$\mathrm{el}$, and in turn, adiponectin increased T-cadherin protein expression in aorta, heart, and muscle in mice $(14,98)$. Thus, both genetically modified mice and human genetic analyses suggest that adiponectin's actions are mediated through T-cadherin.

T-cadherin. T-cadherin is a unique member of the cadherin family of cell adhesion molecules, possessing a GPI anchor instead of a transmembrane and intracellular domain (106) (Figure 1). It is classified as a member of classical cadherins like E-cadherin and $\mathrm{N}$-cadherin by its high homology of 5 extracellular cadherin repeats. However, the GPI anchor on T-cadherin's C-terminus differentiates it from other cadherins (Figure 1).

$\mathrm{T}$-cadherin was cloned by expression cloning from a $\mathrm{C} 2 \mathrm{C} 12$ myotube cDNA library by evaluating cell binding to recombinant adiponectin produced in HEK293 mammalian cells (57). T-cadherin binds multimeric adiponectin, including the hexameric form and larger multimers $(19,57)$. 
Clinical analyses have demonstrated that HMW multimeric adiponectin is the active form, and it exerts various pleiotropic effects (64-67). Recent studies have shown that the active HMW multimers and hexamers of adiponectin accumulate in tissues such as heart, vascular endothelium, and skeletal muscles through interaction with T-cadherin $(14-17,98)$. Plasma adiponectin increased more than 3-fold in T-cadherin-KO mice compared with WT mice, with a dramatic increase of HMW multimeric adiponectin (98). Importantly, several reports have shown the importance of adiponectin association with T-cadherin in organ protection in models of cardiac hypertrophy (14), hind limb ischemia (15), atherosclerosis (16), and skeletal muscle regeneration (17). Furthermore, as discussed above, SNPs near the T-cadherin gene correlate strongly with plasma adiponectin level and cardiovascular disease risk in human subjects $(101,103,105,107-112)$.

T-cadherin is an atypical cadherin with a GPI anchor, and without the transmembrane and intracellular domains required for typical signaling, which has long hindered reaching the conclusion that T-cadherin is the receptor for adiponectin. However, a recent study established a high-affinity interaction between T-cadherin and native HMW multimeric adiponectin purified from mouse serum (19). T-cadherin fused with human IgG Fc as a fusion tag replacing its GPI anchor specifically bound both hexameric and HMW multimeric adiponectin in serum, with a dissociation constant of approximately $1.0 \mathrm{nM}$ and without contribution from other cellular or serum factors (19). The extracellular T-cadherin repeats 1 and 2, which overlap with the regions required for classical cadherin-mediated cell-to-cell adhesion, were critical for the observed adiponectin binding (19) (Figure 1). Moreover, the 130-kDa prodomain-bearing T-cadherin, uniquely expressed on the cell surface among members of the cadherin family, was predominantly increased by adiponectin and significantly contributed to adiponectin binding (19) (Figure 1).

Adiponectin regulates exosome biogenesis and systemic exosome levels. Exosome biogenesis stimulation by adiponectin was the first demonstration that this secreted factor can modulate exosome biogenesis and secretion (18) (Figure 2). Adiponectin was detected in intracellular compartments in endothelial cells of WT mouse aorta by ultrastructural immune-electron microscopy analysis (113). Adiponectin together with T-cadherin accumulated inside multivesicular bodies, the site of exosome generation, both in cultured endothelial cells and in vivo in WT mouse aorta (18). Adiponectin also accumulated in exosomes in the conditioned media of endothelial cells that were cultured with native adiponectin from mouse serum and even in exosomes in the blood of WT mice, but not in T-cadherin-deficient mice (18). The systemic level of exosomes in blood was decreased almost half by genetic loss of adiponectin or of T-cadherin and increased by overexpression of adiponectin in mice (18). Adiponectin existing in serum or purified from serum rich in HMW multimeric adiponectin stimulated exosome production from cultured endothelial cells (18). The exosomal effect of HMW multimeric adiponectin was dose dependent in at least the physiological concentration range (18). Adiponectin-induced increases in exosome biogenesis in cultured cells were dependent on T-cadherin (18), and were not restricted to cultured endothelial cells but also observed in C2C12 differentiated myotubes (17) (Figure 2). Because T-cadherin is expressed broadly in muscle, heart, and vessels, and adiponectin affects systemic exosome levels, the decreased microRNAs in serum exosomes in Dicer-KO mice may be the result of extremely reduced adiponectin and adiponectin-mediated exosome biogenesis in these lipodystrophic mice $(7,18)$.

It has been shown that higher-order oligomerization of protein with membrane anchoring is sufficient for protein sorting into the exosome (114). Furthermore, clustering of an extremely abundant membrane protein, syndecan, via association with the intracellular adaptor syntenin was reported to stimulate exosome biogenesis in a cell culture model (115). Adiponectin, with its multimeric structure, may cause higher-order clustering of T-cadherin, a membrane-anchored protein.

In accordance with the above-mentioned signaling and cellular maintenance roles of exosomes, T-cadherin can transmit adiponectin signals both into T-cadherin-expressing cells, such as endothelial, muscle, and cardiac cells and to neighboring or distant cells by enhancing secretion of exosomes into extracellular fluids and circulation (Figure 2).

Relating to the cellular maintenance role of exosomes on exosome-producing cells, adiponectin stimulated ceramide efflux to exosomes and decreased cellular ceramide levels (18). Ceramide accumulation in cells is known to induce apoptotic pathways $(116,117)$ and attenuate insulin sensitivity $(118,119)$. Disposal of ceramides to macrophages via exosomes may constitute an intercellular and/or interorgan metabolic system of transferring ceramides to macrophages, whose lysosomal functions are much more extensive than other cell types. Overaccumulation of ceramides specifically in macrophages in Farber disease patients who lack lysosomal ceramidase may also indicate the physiological importance of this exosomal ceramide transport to, and degradation in, macrophages (120).

Regarding adiponectin, we return to two important questions mentioned in the Introduction that remain unanswered: (i) Why are such abundant concentrations of adiponectin in plasma required? (ii) Why are large multimeric conformations of adiponectin required for exerting its functions? We suggest that the exosome-stimulating function of adiponectin may partly answer these questions.

\section{Future perspectives}

Technical advances required for studying exosomes' functional role. Currently, technical advances in studies on extracellular vesicle are still lacking, specifically approaches for isolation and discrimination of types of vesicles, i.e., exosomal vesicles of endosomal origin, microvesicles, or ectosomal vesicles of plasma membrane origin, and others such as apoptotic bodies and artifactual particles released from cells broken during experimental processing (121). Lack of sophisticated technology to create loss-of-exosome animals, even restricted to organs or specific types of cells in living animals, limits studies of exosome-mediated processes. For example, inhibition of neutral sphingomyelinase 2 (nSMase2) by the small compound GW4869 is commonly used to block exosome biogenesis. However, nSMase 2 is involved in cellular growth, stress response, and inflammation signaling, and its deficiency causes numerous abnormalities in mice (122). Loss of a specific microRNA will affect its producing cells as well as 
the recipient cells of the exosomal transfer. Measurement of the amounts of exosome in biological fluids is still challenging; light-scattering technologies cannot even discriminate extracellular vesicles from protein aggregates (121), and quantification of total protein or lipid is overestimated as a result of contaminations (121). Technical advances in purification and quantification of exosomes in biological fluids, and in strategies to block exosome production or uptake by recipient cells in animals, will give clearer understanding of the pathophysiological roles of exosomes in metabolic syndrome.

Possible metabolic roles of adiponectin-mediated exosome production. Recent evidence indicates that adiponectin stimulates exosome biogenesis by being endocytosed into endosomes through $\mathrm{T}$-cadherin binding (18). Over 20 years of adiponectin studies suggest that this adipocytokine positively affects insulin sensitivity in addition to providing cardiovascular protection (123-125). Importantly, overexpression of adiponectin in WT mice usually produces stronger effects than adiponectin deficiency. For example, muscle regeneration was improved by adenoviral overexpression of adiponectin, whereas the only tendency toward muscle attenuation was observed in adiponectin-KO mice (17). One important approach may be the application of such adiponectin functions to the development of future medications.
Depending on their source, exosomes are known to influence insulin sensitivity $(5,6,24,126)$ and cardiovascular functions $(127-$ 130). How adiponectin, especially HMW adiponectin, improves insulin sensitivity and cardiac functions through exosomes, and how this pathway can be stimulated for future therapeutics are yet to be investigated. Little has been studied regarding the role of adiponectin mediated through T-cadherin in glucose metabolism and insulin sensitivity in mice. In fact, insulin sensitivity was only evaluated under the chow-diet condition in T-cadherin-KO mice (131). Macrophages are well known to influence inflammation and insulin sensitivity (132-135) and these cells phagocytose the majority of exosomes $(49,51-53)$. Thus, pharmacological strategies to improve the function of the adiponectin/T-cadherin/exosome/macrophage axis may facilitate the reduction of metabolic inflammation and improvement of insulin sensitivity.

Address correspondence to: Shunbun Kita, Department of Metabolic Medicine and Department of Adipose Tissue Management, Graduate School of Medicine, Osaka University, 2-2 Suita, Osaka 565-0871, Japan. Phone: 81.6.6879.3737; Email:shunkita@ endmet.med.osaka-u.ac.jp. Or to: Iichiro Shimomura, Department of Metabolic Medicine, Graduate School of Medicine, Osaka University, 2-2 Suita, Osaka 565-0871, Japan. Phone: 81.6.6879.3744; Email:ichi@endmet.med.osaka-u.ac.jp.
1. Kleinert S, Horton R. Obesity needs to be put into a much wider context. Lancet. 2019;393(10173):724-726.

2. Foreman KJ, et al. Forecasting life expectancy, years of life lost, and all-cause and cause-specific mortality for 250 causes of death: reference and alternative scenarios for 2016-40 for 195 countries and territories. Lancet. 2018;392(10159):2052-2090.

3. Nakamura K, Fuster JJ, Walsh K. Adipokines: a link between obesity and cardiovascular disease. J Cardiol. 2014;63(4):250-259.

4. Flaherty SE, Grijalva A, Xu X, Ables E, Nomani A, Ferrante AW. A lipase-independent pathway of lipid release and immune modulation by adipocytes. Science. 2019;363(6430):989-993.

5. Pan Y, et al. Adipocyte-secreted exosomal microRNA-34a inhibits M2 macrophage polarization to promote obesity-induced adipose inflammation. J Clin Invest. 2019;129(2):834-849.

6. Ying W, et al. Adipose tissue macrophage-derived exosomal miRNAs can modulate in vivo and in vitro insulin sensitivity. Cell.2017;171(2):372-384.e12.

7. Thomou T, et al. Adipose-derived circulating miRNAs regulate gene expression in other tissues. Nature. 2017;542(7642):450-455.

8. Couzin J. Cell biology: The ins and outs of exosomes. Science. 2005;308(5730):1862-1863.

9. Tkach M, Théry C. Communication by extracellular vesicles: where we are and where we need to go. Cell. 2016;164(6):1226-1232.

10. Scherer PE, Williams S, Fogliano M, Baldini G, Lodish HF. A novel serum protein similar to C1q, produced exclusively in adipocytes. J Biol Chem. 1995;270(45):26746-26749.

11. Maeda K, Okubo K, Shimomura I, Funahashi T, Matsuzawa Y, Matsubara K. cDNA cloning and expression of a novel adipose specific collagen-like factor, apM1 (AdiPose Most abundant Gene transcript 1). Biochem Biophys Res Commun.
1996;221(2):286-289.

12. Nakano Y, Tobe T, Choi-Miura NH, Mazda T, Tomita M. Isolation and characterization of GBP28, a novel gelatin-binding protein purified from human plasma. JBiochem. 1996;120(4):803-812.

13. Hu E, Liang P, Spiegelman BM. AdipoQ is a novel adipose-specific gene dysregulated in obesity. J Biol Chem. 1996;271(18):10697-10703.

14. Denzel MS, Scimia MC, Zumstein PM, Walsh K, Ruiz-Lozano P, Ranscht B. T-cadherin is critical for adiponectin-mediated cardioprotection in mice. J Clin Invest. 2010;120(12):4342-4352.

15. Parker-Duffen JL, et al. T-cadherin is essential for adiponectin-mediated revascularization. J Biol Chem. 2013;288(34):24886-24897.

16. Fujishima Y, et al. Adiponectin association with T-cadherin protects against neointima proliferation and atherosclerosis. FASEB J. 2017;31(4):1571-1583.

17. Tanaka Y, et al. Adiponectin promotes muscle regeneration through binding to T-cadherin. $\mathrm{Sci}$ Rep. 2019;9(1):16.

18. Obata Y, et al. Adiponectin/T-cadherin system enhances exosome biogenesis and decreases cellular ceramides by exosomal release. JCI Insight. 2018;3(8):99680.

19. Fukuda S, et al. The unique prodomain of T-cadherin plays a key role in adiponectin binding with the essential extracellular cadherin repeats 1 and 2. JBiol Chem. 2017;292(19):7840-7849.

20. Halberg N, Wernstedt-Asterholm I, Scherer PE. The adipocyte as an endocrine cell. Endocrinol Metab Clin North Am. 2008;37(3):753-768, x-xi.

21. Lehr S, et al. Identification and validation of novel adipokines released from primary human adipocytes. Mol Cell Proteomics. 2012;11(1):M111.010504.

22. Lehr S, Hartwig S, Sell H. Adipokines: a treasure trove for the discovery of biomarkers for metabolic disorders. Proteomics Clin Appl. 2012;6(1-2):91-101.
23. Zhang Y, Proenca R, Maffei M, Barone M, Leopold L, Friedman JM. Positional cloning of the mouse obese gene and its human homologue. Nature. 1994;372(6505):425-432.

24. Crewe C, et al. An Endothelial-to-adipocyte extracellular vesicle axis governed by metabolic state. Cell. 2018;175(3):695-708.e13.

25. Van Vleet ES, Candileri S, McNeillie J, Reinhardt SB, Conkright ME, Zwissler A. Neutral lipid components of eleven species of Caribbean sharks. Comp Biochem Physiol, B. 1984;79(4):549-554.

26. Gesta S, Tseng YH, Kahn CR. Developmental origin of fat: tracking obesity to its source. Cell. 2007;131(2):242-256.

27. Williams RL, Urbé S. The emerging shape of the ESCRT machinery. Nat Rev Mol Cell Biol. 2007;8(5):355-368.

28. Cawthorn WP, et al. Bone marrow adipose tissue is an endocrine organ that contributes to increased circulating adiponectin during caloric restriction. Cell Metab. 2014;20(2):368-375.

29. Horowitz MC, et al. Bone marrow adipocytes. Adipocyte. 2017;6(3):193-204.

30. Huotari J, Helenius A. Endosome maturation. EMBO J. 2011;30(17):3481-3500.

31. Hessvik NP, Llorente A. Current knowledge on exosome biogenesis and release. Cell Mol Life Sci. 2018;75(2):193-208.

32. Santovito D, et al. Plasma exosome microRNA profiling unravels a new potential modulator of adiponectin pathway in diabetes: effect of glycemic control. J Clin Endocrinol Metab. 2014;99(9):E1681-E1685.

33. Zhang $\mathrm{H}$, et al. Serum exosomes mediate delivery of arginase 1 as a novel mechanism for endothelial dysfunction in diabetes. Proc Natl Acad Sci USA. 2018;115(29):E6927-E6936.

34. Chevillet JR, et al. Quantitative and stoichiometric analysis of the microRNA con- 
tent of exosomes. Proc Natl Acad Sci USA. 2014;111(41):14888-14893.

35. Freeman DW, et al. Altered extracellular vesicle concentration, cargo, and function in diabetes. Diabetes. 2018;67(11):2377-2388.

36. Witwer KW, et al. Standardization of sample collection, isolation and analysis methods in extracellular vesicle research. J Extracell Vesicles. 2013;2:20360.

37. Mateescu B, et al. Obstacles and opportunities in the functional analysis of extracellular vesicle RNA - an ISEV position paper. J Extracell Vesicles. 2017;6(1):1286095.

38. Rider MA, Hurwitz SN, Meckes DG. ExtraPEG: A polyethylene glycol-based method for enrichment of extracellular vesicles. Sci Rep. 2016;6:23978.

39. Eitan E, Suire C, Zhang S, Mattson MP. Impact of lysosome status on extracellular vesicle content and release. Ageing Res Rev. 2016;32:65-74.

40. Rajendran L, et al. Alzheimer's disease beta-amyloid peptides are released in association with exosomes. Proc Natl Acad Sci USA. 2006;103(30):11172-11177.

41. Saman S, et al. Exosome-associated tau is secreted in tauopathy models and is selectively phosphorylated in cerebrospinal fluid in early Alzheimer disease. J Biol Chem. 2012;287(6):3842-3849.

42. Fevrier B, et al. Cells release prions in association with exosomes. Proc Natl Acad Sci USA. 2004;101(26):9683-9688.

43. Takahashi A, et al. Exosomes maintain cellular homeostasis by excreting harmful DNA from cells. Nat Commun. 2017;8:15287.

44. Hoshino A, et al. Tumour exosome integrins determine organotropic metastasis. Nature. 2015;527(7578):329-335.

45. Tian T, Wang Y, Wang H, Zhu Z, Xiao Z. Visualizing of the cellular uptake and intracellular trafficking of exosomes by live-cell microscopy. JCell Biochem. 2010;111(2):488-496.

46. Parolini I, et al. Microenvironmental $\mathrm{pH}$ is a key factor for exosome traffic in tumor cells. J Biol Chem. 2009;284(49):34211-34222.

47. Morelli AE, et al. Endocytosis, intracellular sorting, and processing of exosomes by dendritic cells. Blood. 2004;104(10):3257-3266.

48. Miyanishi M, Tada K, Koike M, Uchiyama Y, Kitamura T, Nagata S. Identification of Tim4 as a phosphatidylserine receptor. Nature. 2007;450(7168):435-439.

49. Feng D, et al. Cellular internalization of exosomes occurs through phagocytosis. Traffic. 2010;11(5):675-687.

50. Matsumoto A, et al. Role of phosphatidylserine-derived negative surface charges in the recognition and uptake of intravenously injected B16BL6-derived exosomes by macrophages. JPharm Sci. 2017;106(1):168-175.

51. Imai $\mathrm{T}$, et al. Macrophage-dependent clearance of systemically administered B16BL6-derived exosomes from the blood circulation in mice. JExtracell Vesicles. 2015;4:26238.

52. Takahashi Y, et al. Visualization and in vivo tracking of the exosomes of murine melanoma B16-BL6 cells in mice after intravenous injection. J Biotechnol. 2013;165(2):77-84.

53. Yamashita T, Takahashi Y, Nishikawa M, Takakura Y. Effect of exosome isolation methods on physicochemical properties of exosomes and clearance of exosomes from the blood circulation. Eur J Pharm Biopharm. 2016;98:1-8.

54. Grijalva A, Xu X, Ferrante AW. Autophagy is dispensable for macrophage-mediated lipid homeostasis in adipose tissue. Diabetes. 2016;65(4):967-980.

55 . Yamauchi T, et al. Cloning of adiponectin receptors that mediate antidiabetic metabolic effects. Nature. 2003;423(6941):762-769.

56. Takemura Y, et al. Adiponectin modulates inflammatory reactions via calreticulin receptor-dependent clearance of early apoptotic bodies. J Clin Invest. 2007;117(2):375-386

57. Hug C, Wang J, Ahmad NS, Bogan JS, Tsao TS, Lodish HF. T-cadherin is a receptor for hexameric and high-molecular-weight forms of Acrp30/adiponectin. Proc Natl Acad Sci USA. 2004;101(28):10308-10313.

58. Yamauchi T, et al. Targeted disruption of AdipoR1 and AdipoR2 causes abrogation of adiponectin binding and metabolic actions. Nat Med. 2007;13(3):332-339.

59. Iwabu $\mathrm{M}$, et al. Adiponectin and AdipoR1 regulate PGC-1alpha and mitochondria by $\mathrm{Ca}(2+)$ and AMPK/SIRT1. Nature. 2010;464(7293):1313-1319.

60. Holland WL, et al. Receptor-mediated activation of ceramidase activity initiates the pleiotropic actions of adiponectin. Nat Med.2011;17(1):55-63.

61. Pajvani UB, et al. Structure-function studies of the adipocyte-secreted hormone Acrp30/adiponectin. Implications for metabolic regulation and bioactivity. J Biol Chem. 2003;278(11):9073-9085.

62. Tsao TS, et al. Role of disulfide bonds in Acrp30/ adiponectin structure and signaling specificity. Different oligomers activate different signal transduction pathways. J Biol Chem. 2003;278(50):50810-50817.

63. Waki $\mathrm{H}$, et al. Impaired multimerization of human adiponectin mutants associated with diabetes. Molecular structure and multimer formation of adiponectin. J Biol Chem. 2003;278(41):40352-40363.

64. Hara K, et al. Measurement of the high-molecular weight form of adiponectin in plasma is useful for the prediction of insulin resistance and metabolic syndrome. Diabetes Care. 2006;29(6):1357-1362.

65. Inoue $\mathrm{T}$, et al. High molecular weight adiponectin as a predictor of long-term clinical outcome in patients with coronary artery disease. Am J Cardiol. 2007;100(4):569-574.

66. Hirose H, Yamamoto Y, Seino-Yoshihara Y, Kawabe H, Saito I. Serum high-molecular-weight adiponectin as a marker for the evaluation and care of subjects with metabolic syndrome and related disorders. J Atheroscler Thromb. 2010;17(12):1201-1211.

67. Basu R, Pajvani UB, Rizza RA, Scherer PE. Selective downregulation of the high molecular weight form of adiponectin in hyperinsulinemia and in type 2 diabetes: differential regulation from nondiabetic subjects. Diabetes. 2007;56(8):2174-2177.

68. Koenen TB, et al. Adiponectin multimer distribution in patients with familial combined hyperlipidemia. Biochem Biophys Res Commun. 2008;376(1):164-168.

69. Pajvani UB, et al. Complex distribution, not absolute amount of adiponectin, correlates with thiazolidinedione-mediated improvement in insulin sensitivity. J Biol Chem. 2004;279(13):12152-12162.

70. Suzuki S, Wilson-Kubalek EM, Wert D, Tsao TS, Lee DH. The oligomeric structure of high molecular weight adiponectin. FEBS Lett. 2007;581(5):809-814.

71. Miller NE, et al. Secretion of adipokines by human adipose tissue in vivo: partitioning between capillary and lymphatic transport. Am JPhysiol Endocrinol Metab. 2011;301(4):E659-E667.

72. Gavrila A, Peng CK, Chan JL, Mietus JE, Goldberger AL, Mantzoros CS. Diurnal and ultradian dynamics of serum adiponectin in healthy men: comparison with leptin, circulating soluble leptin receptor, and cortisol patterns. JClin Endocrinol Metab. 2003;88(6):2838-2843.

73. Bogan JS, Lodish HF. Two compartments for insulin-stimulated exocytosis in 3T3-L1 adipocytes defined by endogenous ACRP30 and GLUT4. JCell Biol. 1999;146(3):609-620.

74. Arita Y, et al. Paradoxical decrease of an adipose-specific protein, adiponectin, in obesity. Biochem Biophys Res Commun. 1999;257(1):79-83.

75. Tilg H, Moschen AR. Adipocytokines: mediators linking adipose tissue, inflammation and immunity. Nat Rev Immunol. 2006;6(10):772-783.

76. Cnop M, et al. Relationship of adiponectin to body fat distribution, insulin sensitivity and plasma lipoproteins: evidence for independent roles of age and sex. Diabetologia. 2003;46(4):459-469.

77. Weyer C, et al. Hypoadiponectinemia in obesity and type 2 diabetes: close association with insulin resistance and hyperinsulinemia. JClin Endocrinol Metab. 2001;86(5):1930-1935.

78. Maeda N, et al. PPARgamma ligands increase expression and plasma concentrations of adiponectin, an adipose-derived protein. Diabetes. 2001;50(9):2094-2099.

79. Fasshauer M, et al. Adiponectin gene expression and secretion is inhibited by interleukin- 6 in 3T3-L1 adipocytes. Biochem Biophys Res Commun. 2003;301(4):1045-1050.

80. Furukawa K, et al. Adiponectin down-regulates acyl-coenzyme A:cholesterol acyltransferase- 1 in cultured human monocyte-derived macrophages. Biochem Biophys Res Commun. 2004;317(3):831-836.

81. Kurata A, et al. Blockade of angiotensin II type1 receptor reduces oxidative stress in adipose tissue and ameliorates adipocytokine dysregulation. Kidney Int. 2006;70(10):1717-1724.

82. Nishizawa $\mathrm{H}$, et al. Androgens decrease plasma adiponectin, an insulin-sensitizing adipocyte-derived protein. Diabetes. 2002;51(9):2734-2741.

83. Hirata A, et al. Blockade of mineralocorticoid receptor reverses adipocyte dysfunction and insulin resistance in obese mice. Cardiovasc Res. 2009;84(1):164-172.

84. Hirata A, et al. Contribution of glucocorticoid-mineralocorticoid receptor pathway on the obesity-related adipocyte dysfunction. Biochem Biophys Res Commun. 2012;419(2):182-187.

85. Obata Y, et al. Relationship between serum adiponectin levels and age in healthy subjects and patients with type 2 diabetes. Clin Endocrinol (Oxf). 2013;79(2):204-210.

86. Adamczak M, Rzepka E, Chudek J, Wiecek A. Ageing and plasma adiponectin concentration in apparently healthy males and females. Clin 
Endocrinol (Oxf). 2005;62(1):114-118.

87. Isobe $\mathrm{T}$, et al. Influence of gender, age and renal function on plasma adiponectin level: the Tanno and Sobetsu study. Eur JEndocrinol. 2005;153(1):91-98.

88. Yaturu S, Reddy RD, Rains J, Jain SK. Plasma and urine levels of resistin and adiponectin in chronic kidney disease. Cytokine. 2007;37(1):1-5.

89. Barnea M, Shamay A, Stark AH, Madar Z. A highfat diet has a tissue-specific effect on adiponectin and related enzyme expression. Obesity (Silver Spring). 2006;14(12):2145-2153.

90. Naderali EK, et al. A fat-enriched, glucoseenriched diet markedly attenuates adiponectin mRNA levels in rat epididymal adipose tissue. Clin Sci. 2003;105(4):403-408.

91. Kistorp C, et al. Plasma adiponectin, body mass index, and mortality in patients with chronic heart failure. Circulation. 2005;112(12):1756-1762.

92. George J, et al. Circulating adiponectin concentrations in patients with congestive heart failure. Heart. 2006;92(10):1420-1424.

93. Tsutamoto T, et al. Total and high molecular weight adiponectin, haemodynamics, and mortality in patients with chronic heart failure. Eur Heart J. 2007;28(14):1723-1730.

94. Schnabel R, et al. Association of adiponectin with adverse outcome in coronary artery disease patients: results from the AtheroGene study. Eur Heart J. 2008;29(5):649-657.

95. Tsukamoto $O$, et al. Natriuretic peptides enhance the production of adiponectin in human adipocytes and in patients with chronic heart failure. JAm Coll Cardiol. 2009;53(22):2070-2077.

96. Yamaji M, et al. Effect of carvedilol on plasma adiponectin concentration in patients with chronic heart failure. Circ J. 2009;73(6):1067-1073.

97. Van Berendoncks AM, et al. $\beta$-blockers modify the prognostic value of adiponectin in chronic heart failure. Int J Cardiol. 2011;150(3):296-300.

98. Matsuda K, et al. Positive feedback regulation between adiponectin and T-cadherin impacts adiponectin levels in tissue and plasma of male mice. Endocrinology. 2015;156(3):934-946.

99. Dastani Z, et al. Novel loci for adiponectin levels and their influence on type 2 diabetes and metabolic traits: a multi-ethnic meta-analysis of 45,891 individuals. PLoS Genet. 2012;8(3):e1002607.

100. Yaghootkar H, et al. Mendelian randomization studies do not support a causal role for reduced circulating adiponectin levels in insulin resistance and type 2 diabetes. Diabetes. 2013;62(10):3589-3598.

101. Borges MC, Lawlor DA, de Oliveira C, White J, Horta BL, Barros AJ. Role of adiponectin in coronary heart disease risk: a Mendelian randomization study. Circ Res. 2016;119(3):491-499.

102.Xia JY, et al. Acute loss of adipose tissue-derived adiponectin triggers immediate metabolic deterioration in mice. Diabetologia. 2018;61(4):932-941.

103. Buniello A, et al. The NHGRI-EBI GWAS Catalog of published genome-wide association studies, targeted arrays and summary statistics 2019. Nucleic Acids Res. 2019;47(D1):D1005-D1012.

104.Palmer ND, et al. Genetic variants associated with quantitative glucose homeostasis traits translate to type 2 diabetes in Mexican Americans: The GUARDIAN (Genetics Underlying Diabetes in Hispanics) Consortium. Diabetes.
2015;64(5):1853-1866.

105. Kitamoto A, et al. CDH13 polymorphisms are associated with adiponectin levels and metabolic syndrome traits independently of visceral fat mass. J Atheroscler Thromb. 2016;23(3):309-319.

106.Ranscht B, Dours-Zimmermann MT. T-cadherin, a novel cadherin cell adhesion molecule in the nervous system lacks the conserved cytoplasmic region. Neuron. 1991;7(3):391-402.

107. Chung CM, et al. A genome-wide association study reveals a quantitative trait locus of adiponectin on $\mathrm{CDH} 13$ that predicts cardiometabolic outcomes. Diabetes. 2011;60(9):2417-2423.

108. Fava C, et al. A variant upstream of the $\mathrm{CDH} 13$ adiponectin receptor gene and metabolic syndrome in Swedes. Am JCardiol. 2011;108(10):1432-1437.

109. Gao H, et al. Genetic variation in CDH13 is associated with lower plasma adiponectin levels but greater adiponectin sensitivity in East Asian populations. Diabetes. 2013;62(12):4277-4283.

110. Jee SH, et al. Adiponectin concentrations: a genome-wide association study. Am J Hum Genet. 2010;87(4):545-552.

111. Wu Y, et al. Genome-wide association study for adiponectin levels in Filipino women identifies $\mathrm{CDH} 13$ and a novel uncommon haplotype at KNG1-ADIPOQ. Hum Mol Genet. 2010;19(24):4955-4964.

112. Morisaki H, et al. CDH13 gene coding T-cadherin influences variations in plasma adiponectin levels in the Japanese population. Hum Mutat. 2012;33(2):402-410.

113. Mori T, et al. Ultrastructural localization of adiponectin protein in vasculature of normal and atherosclerotic mice. Sci Rep. 2014;4:4895.

114. Fang Y, Wu N, Gan X, Yan W, Morrell JC, Gould SJ. Higher-order oligomerization targets plasma membrane proteins and HIV gag to exosomes. PLoS Biol. 2007;5(6):e158.

115. Baietti MF, et al. Syndecan-syntenin-ALIX regulates the biogenesis of exosomes. Nat Cell Biol. 2012;14(7):677-685.

116. Niaudet $C$, et al. Plasma membrane reorganization links acid sphingomyelinase/ceramide to p38 MAPK pathways in endothelial cells apoptosis. Cell Signal. 2017;33:10-21.

117. Yabu T, et al. Stress-induced ceramide generation and apoptosis via the phosphorylation and activation of nSMase1 by JNK signaling. Cell Death Differ. 2015;22(2):258-273.

118. Xia JY, et al. Targeted induction of ceramide degradation leads to improved systemic metabolism and reduced hepatic steatosis. Cell Metab. 2015;22(2):266-278.

119. Chaurasia B, Summers SA. Ceramides - lipotoxic inducers of metabolic disorders: (Trends in Endocrinology and Metabolism 26, 538-550; 2015). Trends Endocrinol Metab. 2018;29(1):66-67.

120. Yeager AM, Uhas KA, Coles CD, Davis PC, Krause WL, Moser HW. Bone marrow transplantation for infantile ceramidase deficiency (Farber disease). Bone Marrow Transplant. 2000;26(3):357-363.

121. Théry C, et al. Minimal information for studies of extracellular vesicles 2018 (MISEV2018): a position statement of the International Society for Extracellular Vesicles and update of the MISEV2014 guidelines. J Extracell Vesicles.
2018;7(1):1535750.

122.Wu BX, Clarke CJ, Hannun YA. Mammalian neutral sphingomyelinases: regulation and roles in cell signaling responses. Neuromolecular Med. 2010;12(4):320-330.

123. Yamauchi T, et al. The fat-derived hormone adiponectin reverses insulin resistance associated with both lipoatrophy and obesity. Nat Med. 2001;7(8):941-946.

124.Berg AH, Combs TP, Du X, Brownlee M, Scherer PE. The adipocyte-secreted protein Acrp30 enhances hepatic insulin action. Nat Med. 2001;7(8):947-953.

125. Maeda N, et al. Diet-induced insulin resistance in mice lacking adiponectin/ACRP30. Nat Med. 2002;8(7):731-737.

126.Zhao H, et al. Exosomes from adipose-derived stem cells attenuate adipose inflammation and obesity through polarizing M2 macrophages and beiging in white adipose tissue. Diabetes. 2018;67(2):235-247.

127. Lai RC, et al. Exosome secreted by MSC reduces myocardial ischemia/reperfusion injury. Stem Cell Res. 2010;4(3):214-222.

128. Arslan F, et al. Mesenchymal stem cell-derived exosomes increase ATP levels, decrease oxidative stress and activate PI3K/Akt pathway to enhance myocardial viability and prevent adverse remodeling after myocardial ischemia/reperfusion injury. Stem Cell Res. 2013;10(3):301-312.

129. Lee C, et al. Exosomes mediate the cytoprotective action of mesenchymal stromal cells on hypoxia-induced pulmonary hypertension. Circulation. 2012;126(22):2601-2611.

130. Barile L, et al. Extracellular vesicles from human cardiac progenitor cells inhibit cardiomyocyte apoptosis and improve cardiac function after myocardial infarction. Cardiovasc Res. 2014;103(4):530-541.

131. Tyrberg B, et al. T-cadherin (Cdh13) in association with pancreatic $\beta$-cell granules contributes to second phase insulin secretion. Islets. 2011;3(6):327-337.

132. Olefsky JM, Glass CK. Macrophages, inflammation, and insulin resistance. Annu Rev Physiol. 2010;72:219-246.

133. Hotamisligil GS. Inflammation and metabolic disorders. Nature. 2006;444(7121):860-867.

134. Chawla A, Nguyen KD, Goh YP. Macrophagemediated inflammation in metabolic disease. Nat Rev Immunol. 2011;11(11):738-749.

135. Saltiel AR, Olefsky JM. Inflammatory mechanisms linking obesity and metabolic disease. JClin Invest. 2017;127(1):1-4.

136. Mak HY. Lipid droplets as fat storage organelles in Caenorhabditis elegans: Thematic Review Series: Lipid droplet synthesis and metabolism: from yeast to man. JLipid Res. 2012;53(1):28-33.

137. Gao Q, et al. Pet1Op is a yeast perilipin that stabilizes lipid droplets and promotes their assembly. JCell Biol. 2017;216(10):3199-3217.

138. Chughtai AA, et al. Perilipin-related protein regulates lipid metabolism in C. elegans. PeerJ. 2015;3:e1213.

139. Kajimura D, et al. Adiponectin regulates bone mass via opposite central and peripheral mechanisms through FoxO1. Cell Metab. 2013;17(6):901-915. 